\title{
Four-wave mixing with multi-color laser in extreme ultraviolet region
}

\section{Thong Huy Chau, Khuong Ba Dinh, Khoa Anh Tran, Peter Hannaford, Lap Van Dao}

Thong Huy Chau, Khuong Ba Dinh, Khoa Anh Tran, Peter Hannaford, Lap Van Dao, "Four-wave mixing with multi-color laser in extreme ultraviolet region," Proc. SPIE 11886, International Conference on X-Ray Lasers 2020, 118860F (8 July 2021); doi: 10.1117/12.2592811 


\title{
Four-wave mixing with multi-color laser in extreme ultraviolet region
}

\author{
Thong Huy Chau ${ }^{* 1}$, Khuong Ba Dinh², Khoa Anh Tran ${ }^{1}$, Peter Hannaford ${ }^{1}$, and \\ Lap Van Dao ${ }^{1}$ \\ ${ }^{1}$ Optical Sciences Centre, Swinburne University of Technology, Melbourne 3122, Australia. \\ ${ }^{2}$ Advanced Institute of Science and Technology, The University of Danang, Danang, Vietnam \\ *thongchau@swin.edu.au; phone+61392145685
}

\begin{abstract}
Studies of the generation and propagation of light fields in the extreme ultraviolet (XUV) can provide insights into the fundamental interaction of atoms in highly excited levels and ionized atoms. In this paper, we present experimental results of nonlinear four-wave mixing (FWM) processes using a combination of XUV radiation and optical pulses in argon gas. The XUV pulses are produced by phase-matched high-order harmonic generation (HHG). Optimized phase-matching of collinear multiple-cycle laser pulses with incommensurate frequencies $(800 \mathrm{~nm}, 1400 \mathrm{~nm}$, and $560 \mathrm{~nm})$ is used to indicate the different pathways of the third-order and fifth-order nonlinear responses in the mixing process in a single gas cell configuration. A perturbative nonlinear optics approach can be used to explain our cascaded wave-mixing patterns. Our results reveal that the time-dependent spectral features of the mixing fields are associated with auto-ionization processes. Overall, the intensity and frequency modulation of the wave-mixing fields provides a new technique to investigate the dynamical evolution of electron wave-packets in atomic and molecular gases.
\end{abstract}

Keywords: Extreme ultraviolet, four-wave mixing, cascaded perturbative nonlinear optics, electron wave-packets, argon, dynamics.

\section{INTRODUCTION}

With the availability of XUV light sources for spectroscopic techniques, the ability to be able to tune and select regions of interest in XUV bands is especially significant. A variety of methods such as multilayer mirror ${ }^{1}$, time-delay compensated monochromator ${ }^{2}$, or opto-optical modulation ${ }^{3}$ have been used but these cause losses in a large fraction of the XUV. Fourwave mixing (FWM) spectroscopy in the XUV region stands out from other contemporary techniques not only because it allows tuning of the XUV radiation but also because it is extremely useful for studying time-resolved dynamics of atomic and molecular systems. Together with other methods, including ultrafast photoelectron spectroscopy ${ }^{4}, \mathrm{XUV}$ femtosecond and attosecond transient absorption spectroscopy ${ }^{5}$ and reaction- microscope methods ${ }^{6}$, XUV-FWM spectroscopy can provide information on the dynamics of background-free electron wave-packets which can be beneficial for the analysis of quantum beat interference patterns arising from different quantum pathways ${ }^{7,8}$.

To create a source for XUV-FWM spectroscopy, gas-phase high-harmonic generation (HHG) can be used to provide an attosecond pulse train light source in our experiments. Recently, other studies have investigated different media for both solid-state and liquid-state HHG. However, the high cut-off energy reaches a limit due to the restriction of the damage threshold. The maximum harmonic energy that can be achieved is currently around $40 \mathrm{eV}^{9}$ and $20 \mathrm{eV}^{10}$ for solid-state HHG and liquid-state HHG, respectively. Another method that has recently achieved hundred-gigawatt-scale of attosecond $\mathrm{X}$-ray pulses is the free-electron laser using the Linac Coherent Light Source ${ }^{11}$. However, this method requires a massivescale laboratory, whereas HHG is a compact system which can be conducted in a small-size laboratory.

In our table-top system, we optimize the macroscopic phase-matching for HHG and the phase-matched cascaded XUVFWM in a single gas cell experimental configuration. An attosecond pulse train consisting of an odd-harmonic spectral comb is generated, which repeats at twice the optical cycle of the fundamental driving field $(800 \mathrm{~nm})$. To overcome the problem of a fixed up-conversion and the low efficiency of HHG on the order of $\left(\lambda^{-6}\right)^{12}$ we introduce an additional collinear control beam to initialize the cascaded wave-mixing process. In this way, we can enrich the XUV spectrum and tune the wavelength to increase the flexibility of the original HHG spectrum.

International Conference on X-Ray Lasers 2020, edited by Davide Bleiner,

Proc. of SPIE Vol. 11886, 118860F · (C) 2021 SPIE · CCC code:

0277-786X/21/\$21 - doi: $10.1117 / 12.2592811$ 
The combination of an attosecond pulse train and cascaded wave-mixing in the XUV region provides a novel tool to access the electron dynamics of the interaction of atoms and molecules with intense laser fields ${ }^{13}$. Moreover, all of the interactions occurring in our single gas cell chamber allows us to simplify the experimental setup while still being able to probe the time-evolution of the spectral shape of the electron wave-packet in the XUV cascaded wave-mixing process.

In our experiment, we optimize the sequence of the multi-color, multi-cycle laser field (wavelengths $1400 \mathrm{~nm}, 800 \mathrm{~nm}$, $560 \mathrm{~nm}$ ) with the XUV beam to induce a nonlinear parametric conversion process in an atomic gas of argon. A coherent XUV mixing field is accumulated in our experiment as a result of the FWM process, in which the third and fifth-order of the perturbative nonlinear response is taken into account ${ }^{14-16}$. We demonstrate the utility of the multi-color FWM technique to generate a narrow-bandwidth XUV light source. This cannot be accessed by simple HHG generation and its coherence is better than single-color FWM. Also, the time-evolution and information about the dynamics of the interaction process can be obtained by providing a time-delay between the laser pulses in the FWM setup.

\section{EXPERIMENTAL SETUP}

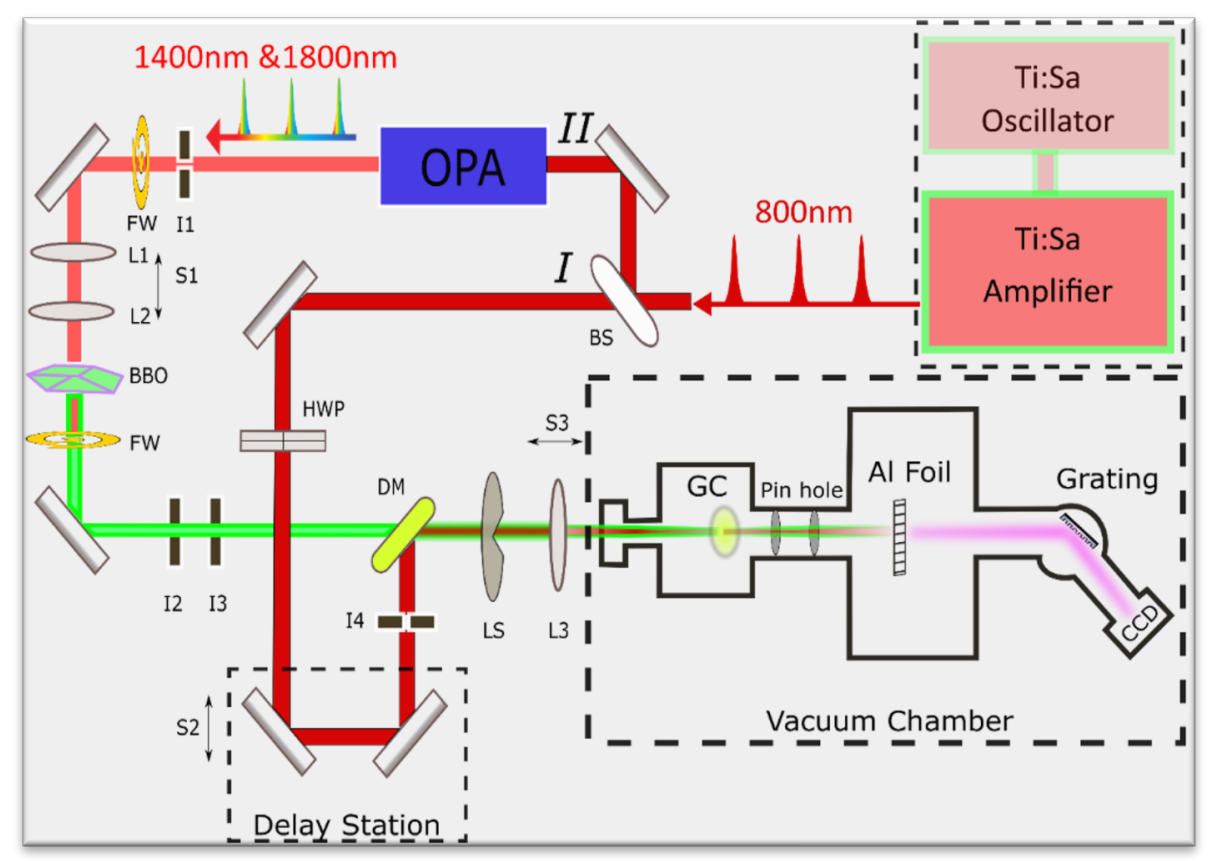

Figure 1: Experimental setup

For the pump-probe spectroscopy configuration, we split an optimized $800 \mathrm{~nm}, 6 \mathrm{~mJ}, 30 \mathrm{fs}$ multi-cycle laser beam into two parts with a beam splitter (BS), hereafter called the driving beam (4 mJ - path I) and the control beam (2 mJ - path II). The driving beam of $30 \mathrm{fs}$ duration is passed through a half-wave plate to adjust the polarization of the beam without significant influence on the pulse peak intensity. The delay station in the driving beam path provides translation steps of $0.2 \mathrm{fs}$ relative to the control beam. The iris (I4) is used to optimize the beam intensity on the order of $10^{14} \mathrm{~W} / \mathrm{cm}^{2}$ which is optimal to create HHG in our experiment. The control beam is directed through the optical parametric amplifier (OPA) to create perpendicularly polarized signal $(1400 \mathrm{~nm})$ and idler $(1865 \mathrm{~nm})$ beams, with $40 \mathrm{fs}$ duration. Along the beam, a Beta Barium Borate (BBO) nonlinear crystal, type II, provides an additional color $(560 \mathrm{~nm})$ which is the sum frequency of $800 \mathrm{~nm}$ and $1865 \mathrm{~nm}$. The intensity of the control beam is maintained around $10^{13} \mathrm{~W} / \mathrm{cm}^{2}$ to ensure it does not create any other HHG spectrum and only plays the role of a perturbation field in our experiment.

The multi-color wave-mixing experiment is performed by varying the frequency of the control beam using a pair of filter wheels (FS) to filter out any unwanted frequencies. The driving beam and control beam are subsequently combined into the gas cell (GC) by a dichroic mirror (DM). The laser shutter (LS) located in front of the gas cell is used to control the exposure time for our time-integrated signal intensity measurement. The fine adjustment for the phase-matching condition is made using the pair of telescope lenses L1, L2; the focusing lens L3, the irises I1, I2, I3; and the delay station S2. In which, the pair of lenses L1, L2 can be used to collimate the control beam and to adjust the focal point for the best 
overlapping with the focal point of the driving beam. The pair of irises I1, I2 is used to control the intensity and transverse mode in the control beam and align it for the collinear propagation with the driving beam.

The heart of our experiment is the gas cell which has a chosen length relative to the focal length of the lens L3. In this experiment, the gas cell is $150 \mathrm{~mm}$ long compared to the focal length of $300 \mathrm{~mm}$. The interaction length of the laser in the nonlinear medium is roughly $15 \mathrm{~mm}$, which is much shorter than the length of the gas cell. In order to work with the XUV spectrum generated by the HHG process, a vacuum on the order of $10^{-3}$ to $10^{-5}$ Torr is maintained. A pressure differential between the gas chamber and the vacuum chamber is maintained by introducing two pin-holes which also play a crucial role in alignment of the emission beams. The pin-hole pair then guides the generated spectrum through to the grating and is detected by a charge-coupled device (CCD) camera for spectral analysis.

\section{RESULTS AND DISCUSSION}

\subsection{Generation and phase-matched HHG and FWM}

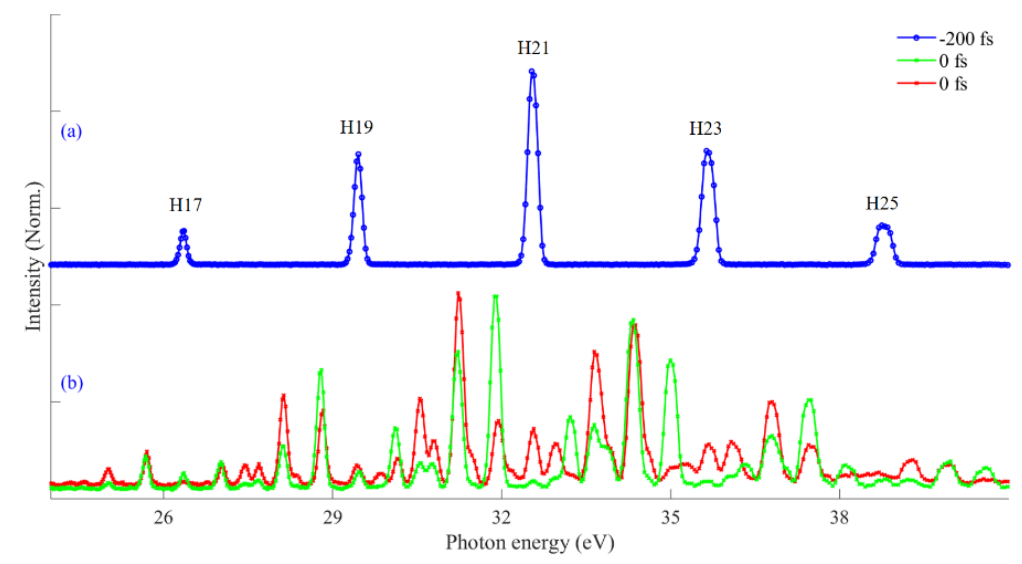

Figure 2: HHG spectrum with 49 Torr of argon. The intensity is normalized to the maximum in each spectrum. (a) HHG spectrum for far negative delay -200 fs, (b) FWM spectrum for phase-matched zero delay $0 \mathrm{fs}$ for cases with/without the presence of $560 \mathrm{~nm}$ corresponding to the color spectrum (green/red).

For a far negative delay (-200 fs), the driving beam arrives before the control beam, and we obtain only the HHG spectrum driven by the $800 \mathrm{~nm}$ beam. Figure 2(a) shows the odd-harmonic spectrum which exhibits a spectral comb structure optimized around the phase-matching condition for harmonic $\mathrm{H} 21(32.55 \mathrm{eV})$. The full spectral linewidth of each harmonic is optimized around $500 \mathrm{meV}$.

The physics underlying the signature spectrum of HHG has been successfully explained using the semi-classical three-step model $^{17}$, the semi-analytical quantum mechanics model ${ }^{18}$ and the fully quantum theory of extreme nonlinear optics ${ }^{19}$. In order to obtain an odd-harmonic spectrum, all the emitted photons have to add constructively together at the moment the electrons recollide back to the parent ion. Calling the fundamental driving laser frequency $\omega_{800}$, the phase-matching for the $\mathrm{q}^{\text {th }}$ harmonic $\left(\omega_{\mathrm{q}}=\mathrm{q} \omega_{800}\right)$ must satisfy the wave-vector mismatch between the driving field and the harmonic field: $\Delta \mathrm{k}_{\mathrm{q}}=\mathrm{qk}_{\left(\omega_{800}\right)}-\mathrm{k}_{\left(\omega_{\mathrm{q}}\right)}=\Delta \mathrm{k}_{\text {neutral }}+\Delta \mathrm{k}_{\text {plasma }}+\Delta \mathrm{k}_{\text {geom }}+\Delta \mathrm{k}_{\text {dipole }} \approx 0$.

The dispersion factor $\Delta \mathrm{k}_{\text {neutral }}$ arises from the neutral gas density, while $\Delta \mathrm{k}_{\text {plasma }}$ is due to the free electron cloud from the ionic gas which is unavoidable during the HHG process. To control these two factors, we optimize the effective focus intensity of the driving field to around $2 \times 10^{14} \mathrm{~W} / \mathrm{cm}^{2}$ to maintain a low ionization rate around a critical value of $3 \%{ }^{20,21}$. In this way, we can maintain a balanced contribution of positive and negative dispersion of the neutral gas and free-electron cloud within the interaction length. Moreover, the effect of free charges on the propagation of the driving field and the XUV field is negligible at this moderate ionization rate ${ }^{22}$.

In addition, the optimal aperture size of iris I4 can be finely adjusted to around 2 to $3 \mathrm{~mm}$, which corresponds to a variable Rayleigh length of approximately 10 to $20 \mathrm{~mm}$ when used with a lens $\mathrm{L} 3$ of focal length $30 \mathrm{~cm}$. Thus, the Rayleigh length is much longer than the effective interaction length $(2 \mathrm{~mm}$ to $5 \mathrm{~mm})$, so that the contribution of the intensity-dependent harmonic dipole phase shift $\Delta \mathrm{k}_{\text {dipole }}$ and the geometric-configuration Gouy phase shift $\Delta \mathrm{k}_{\text {geom }}$ can be neglected ${ }^{22,23}$. The effective interaction length ( $2 \mathrm{~mm}$ to $5 \mathrm{~mm}$ ), i.e., the displacement of focusing point toward the exit pinhole inside the gas 
cell, for phase-matched HHG has been demonstrated in our previous studies ${ }^{24}$ by the observation of the HHG intensity varying quadratically with gas pressure and interaction length.

For a phase-matched zero delay ( $0 \mathrm{fs})$, the control field overlaps with the driving field, and additional frequencies showing four-wave mixing peaks are generated on both sides of each harmonic as shown in Figure 2(b). The enrichment of the spectrum signal can be understood as a perturbative cascaded wave-mixing process ${ }^{16}$. In our experiment, we name as follows: wave-vectors $\mathrm{k}_{800}$ and $\mathrm{k}_{\mathrm{XUV}}$ for the $800 \mathrm{~nm}$ beam and the $\mathrm{q}^{\text {th }}$ harmonic, respectively, $\mathrm{k}_{\mathrm{Ctrl}}$ for the control field for which we can change frequency, and $\mathrm{k}_{\mathrm{MIX}}$ for a new frequency generated by the wave-mixing process. Since the coherence property of the driving field is transferred to the XUV pulse while the contribution of the neutral and plasma dispersion is small for the XUV pulse ${ }^{22}$, the phase-matching condition for the generation of the wave-mixing field within the coherence length of the $\mathrm{q}^{\text {th }}$ harmonic is $\Delta \mathrm{k}=\mathrm{k}_{\mathrm{MIX}}-\left(\mathrm{k}_{\mathrm{XUV}} \pm\left(\mathrm{k}_{800}-\mathrm{k}_{\mathrm{Ctrl}}\right) \approx 0\right.$. The frequency of the mixing field is determined by the law of parity, momentum, and energy conservation in the perturbative wave-mixing regime ${ }^{15,25-28}$.

$$
\omega_{\text {mix }} \approx \omega_{\mathrm{Xuv}} \pm \mathrm{m}\left(\omega_{800}-\omega_{\mathrm{Ctrl}}\right) \approx \mathrm{q} \omega_{800} \pm \mathrm{m} \Delta \omega \text {. }
$$

As a result, the sum and difference frequency mixing frequencies on both sides of the $\mathrm{q}^{\text {th }}$ harmonic in the XUV region are generated in our experiment. The factor $\mathrm{m}=1,2$ corresponds to the third and fifth-order nonlinear wave-mixing process, which is displayed as two peaks on each side of the main XUV harmonic. The difference energy of $0.67 \mathrm{eV}$ corresponds to the difference frequency $\Delta \omega=\omega_{800}-\omega_{\mathrm{Ctrl}}$.

From our previous studies the effective interaction length for the FWM process is identified as about $2 \mathrm{~mm}$ before the exit pinhole inside the gas cell ${ }^{28}$. Within this effective interaction length, the mixing field intensity scales quadratically with gas pressure and interaction length. Plus, the phase effect acting on all component fields is negligible since the ionization rate of the argon gas is kept well below $3 \%$ in our dispersive interactive medium ${ }^{22}$.

\subsection{Studies of multi-color FWM in XUV region}

In this experiment we focus on the influence of the multi-color fields on the modulation of the spectrum without considering complicated energy transitions between resonance states on the background of the continuum of states. Accordingly, we focus on the perturbation nonlinear wave-mixing process with two different FWM processes with and without the presence of the additional field $560 \mathrm{~nm}$ around harmonic $\mathrm{H} 21$.
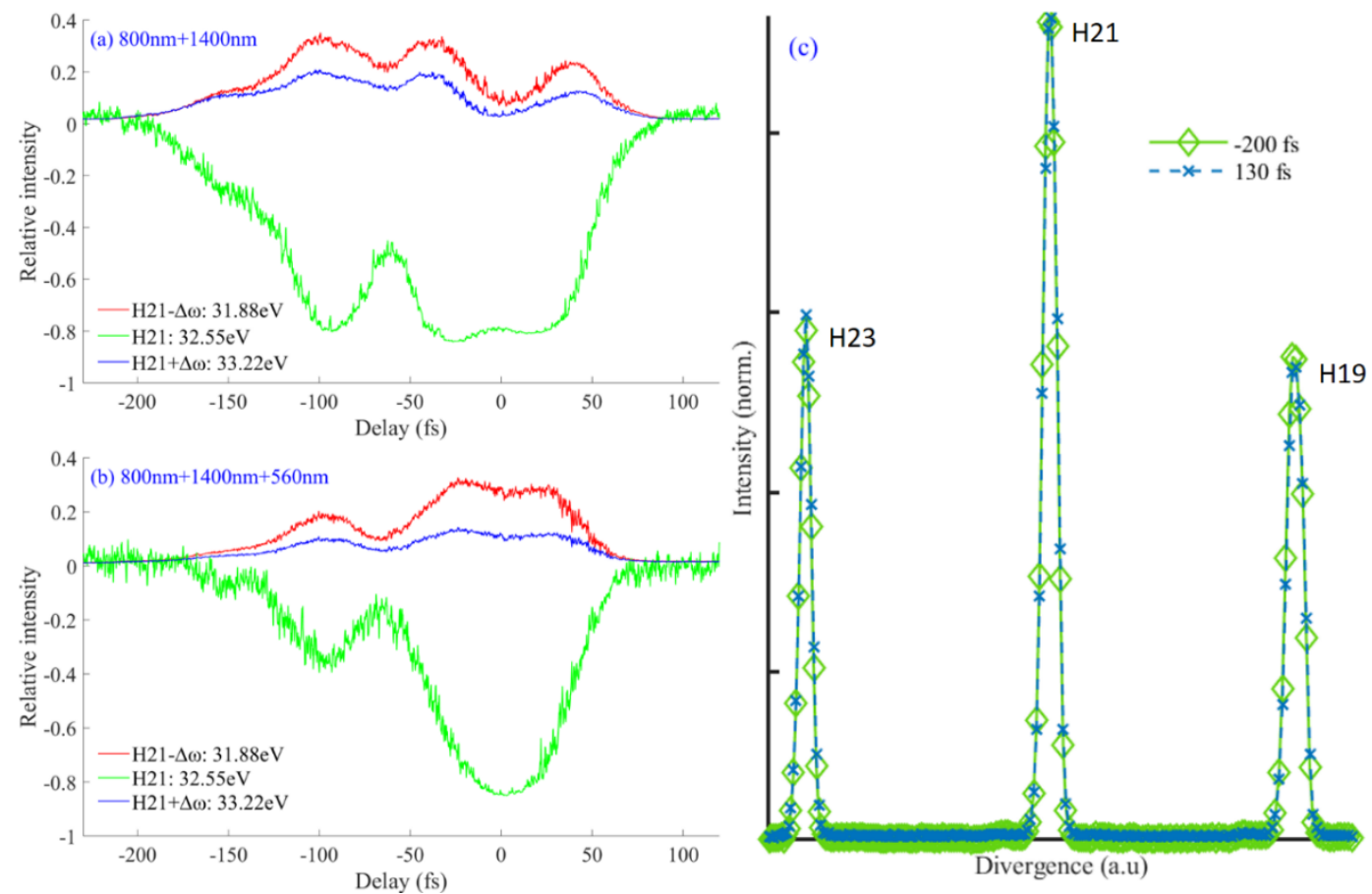

Figure 3: Intensity of XUV radiation versus time-delay at harmonic H21 and its sum and difference frequency, (a) FWM of three input beams of H21, $800 \mathrm{~nm}$ and $1400 \mathrm{~nm}$, (b) FWM of four input beams of H21, $800 \mathrm{~nm}, 1400 \mathrm{~nm}$ and $560 \mathrm{~nm}$, (c) variation of main harmonic spectrum at $\mathrm{H} 19, \mathrm{H} 21, \mathrm{H} 23$ for far-negative and positive delay. 
To ensure the reliability of the data, we monitor the spectrum variation based on the standard odd-harmonic spectrum of HHG. Figure 3(c) shows the sharp feature of HHG peaks which is maintained in our experiment for a far-negative and positive delay. This demonstrates the stability of the driving laser and the HHG generation process before and after the perturbation of the control field.

At zero delay, we have a maximum population depletion of the main harmonic (H21). Figure 3(a) shows the single-color FWM process around harmonic $\mathrm{H} 21(\mathrm{H} 21,800 \mathrm{~nm}$, and $1400 \mathrm{~nm})$, in which we have the energy of the sum frequency $\mathrm{E}_{\mathrm{sum}}=\mathrm{E}_{\mathrm{H} 21}+\left(\mathrm{E}_{800}-\mathrm{E}_{1400}\right)=33.22 \mathrm{eV}$ and the difference frequency $\mathrm{E}_{\text {diff }}=\mathrm{E}_{\mathrm{H} 21}-\left(\mathrm{E}_{800}-\mathrm{E}_{1400}\right)=31.88 \mathrm{eV}$. The phasematched window for this case falls within the range of 100 fs. Figure 3(b) shows the two-color FWM process around harmonic $\mathrm{H} 21(\mathrm{H} 21,800 \mathrm{~nm}, 1400 \mathrm{~nm}$, and $560 \mathrm{~nm}$ ). We observe similar peaks for the sum and difference frequencies but with a different intensity modulation along the time-delay axis. With the additional field $560 \mathrm{~nm}$, the FWM process occurs with a smaller phase-matched window but with higher efficiency for the population transfer from $\mathrm{H} 21$ to first-order mixing frequencies.

To understand the population oscillation among different radiation states within this cascaded wave-mixing process, we interpret the phenomena as follows. Within our single gas cell interaction chamber, the effective interaction length of the HHG process is about $2-5 \mathrm{~mm}$ while the generation of the FWM is about $2 \mathrm{~mm}$ before the exit pinhole ${ }^{28}$. Therefore, the cascaded wave-mixing process can be analyzed in two processes. First, the intense driving field of $\mathrm{k}_{800}$ initiates the HHG process in the argon gas medium. The result is a coherent constructive interference of the emitted XUV radiation which exhibits high temporal and spatial coherence, inherited from $\mathrm{k}_{800}$. Therefore, the synthesized phase-locked XUV pulse and the $800 \mathrm{~nm}$ pulse create a dipole moment and give rise a macroscopic polarization, i.e., addressing the electron wavepacket in a highly excited state. Consequently, if there is no additional effect taking place in this process, the induced electric field of the highly excited atomic gas will cancel the initial field and cause an absorption process. This process will exhibit a characteristic dephasing time which can be recorded by attosecond transient absorption spectroscopy ${ }^{29}$. Second, along the propagation length of $\mathrm{k}_{\mathrm{XUV}}$ and $\mathrm{k}_{800}$, we introduce a perturbation of the control field $\mathrm{k}_{\mathrm{Ctrl}}$ with moderate intensity and parallel polarization to alter the dynamics of the prepared electron wave-packet. For the phase-matching condition, all component fields combine to induce a third-order and fifth-order nonlinear response in the interactive medium. The new generated mixing frequencies enrich our spectrum in the XUV region and this observation indicates the correlation physics with perturbation nonlinear optics theory ${ }^{15,16}$.

When we introduce a time-delay in the control beam, this will result in a time-varying cascaded wave-mixing process which creates a population oscillation among different radiation states at different time-delays as illustrated in Figure 3 and Figure 4. The dynamical process in this nonlinear interaction regime can be extracted to find the correlation of the time-delay dependence between the intrinsic oscillating dipole moment of the medium and the external manipulating electric field. We note that in our recent studies a frequency shift of the FWM field was identified when we analyzed the coupling state of the free electron wave-packet, in which we have a superposition of the continuum and a discrete real excited state of atomic krypton around harmonic H17 $(26.3 \mathrm{eV})^{30}$ and atomic argon around harmonic H19 $(29.3 \mathrm{eV})^{31}$.

In the present experiment, we focus our analysis on the spectrum around harmonic $\mathrm{H} 21$ which lies outside the resonance frequency of argon. From this, we consider the influence of the multi-color fields on the modulation of the XUV spectrum without concern about the resonance of single electron excitation states participating in the dynamics of our system. The energy diagram as illustrated in Figure 4(c) explains the reason for the maximum population transfer in the case of twocolor FWM. 

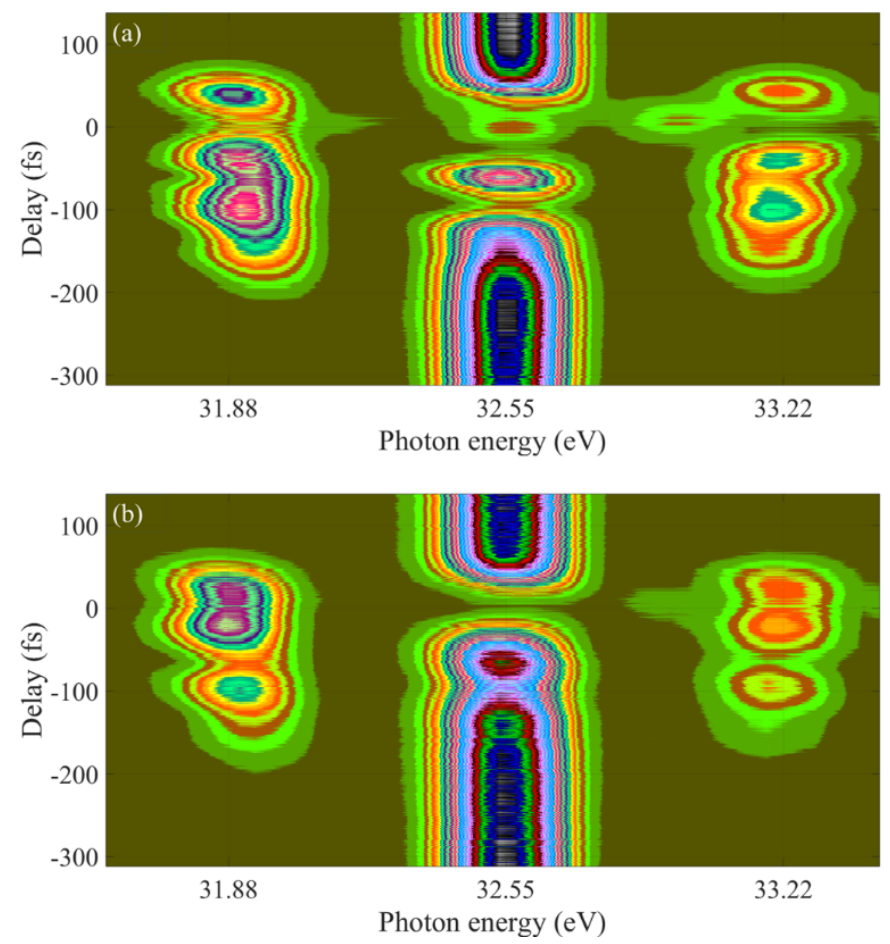

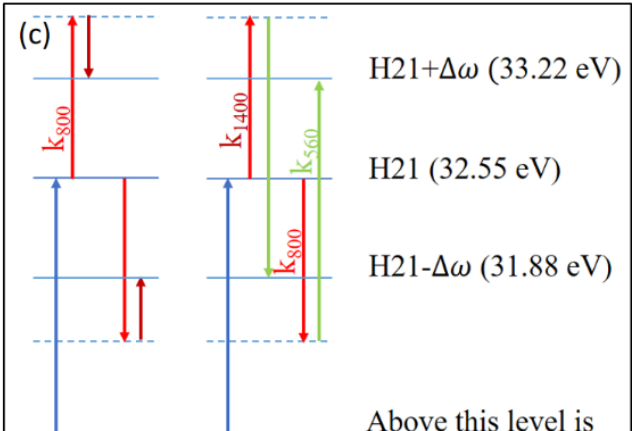

continuum of states $(29.22 \mathrm{eV})$

First ionization threshold $(15.76 \mathrm{eV})$

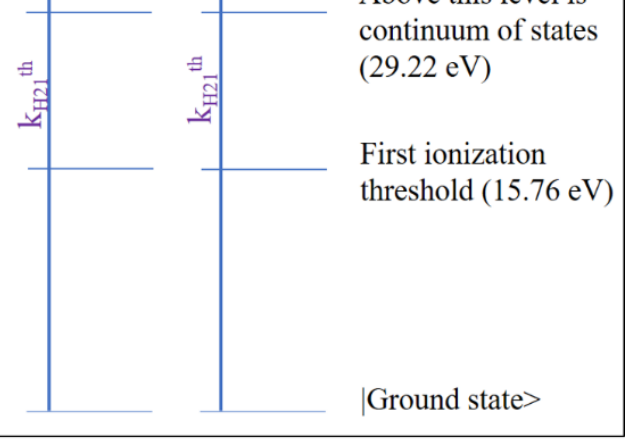

Figure 4: Cascaded FWM spectrum versus time-delay around harmonic H21 (32.55 eV). The sum and difference frequencies appear on the right and left side of H21 corresponding to energies of $33.22 \mathrm{eV}$ and $32.88 \mathrm{eV}$. (a) FWM without the presence of $560 \mathrm{~nm}$, (b) FWM with the presence of $560 \mathrm{~nm}$, (c) energy diagram for FWM process around H21.

The figure illustrates two FWM processes. Interestingly, when we combine the two-color fields of $\mathrm{k}_{1400}$ and $\mathrm{k}_{560}$ in the FWM process, the sum-frequency generation process in the case of FWM with $1400 \mathrm{~nm}$ ends up at the same energy level as with the difference-frequency generation of FWM with $560 \mathrm{~nm}$. Similarly, the difference-frequency generation of the FWM with $1400 \mathrm{~nm}$ ends up at the same energy level as the sum-frequency generation of the FWM with $560 \mathrm{~nm}$.

The above result demonstrates the very high efficiency of the sum and difference frequency generation processes. The contour Figure 4(b) illustrates the wave-mixing process and shows a clear depletion of the main harmonic $\mathrm{H} 21$ at 0 fs in the case of two-color FWM. This phenomenon is in contrast with the single-color FWM result in Figure 4(a) where we observe an unstable intensity oscillation and a sideband oscillation on the sum-frequency side. This result demonstrates the power of the multi-color field FWM process which not only amplifies the new frequency generation process but also produces a higher flux of coherent electron wave-packets. Such a result will be significant for time-resolved studies of electron wave-packet dynamics in highly excited states and in the above-threshold ionization regime. Additionally, the generated wave-packets are background-free and are produced by a perturbative nonlinear wave-mixing process which does not require a dipole-allowed transition to reach the radiation states. As a result of the influence of $\mathrm{H} 21$ on the atomic system in this experiment, we acknowledge that the XUV radiation of H21 $(32.55 \mathrm{eV})$ lies above the first ionization threshold of argon $(15.76 \mathrm{eV})$ and this could lead to either direct ionization or autoionization to the continuum of high Rydberg states in the one-electron excitation system. The higher frequency generation can even exceed the second ionization threshold and become involved in even more complex situations with two or more electron excitation processes.

\section{CONCLUSION}

In this experiment, we have used a single gas cell of argon to perform cascaded four-wave mixing with a multicolor laser field (see also Sects. 2-3 [32]). The results demonstrate the efficiency of the process to generate a coherent electron wave-packet with sum and difference frequencies when we couple two FWM processes together by adding an appropriate color field to the high-order perturbative nonlinear wave-mixing process. In future, the coherent background-free wave-packet generated in our experiment could be tuned to various XUV regions to study the coupling between dark states and bright states in the 
configuration interaction of a quantum system to reveal the dynamics of the time-evolution of the electron wave-packet of several atomic and molecular systems.

\section{REFERENCES}

[1] M. Hatayama, S. Ichimaru, T. Ohcni et al., "Wide-range narrowband multilayer mirror for selecting a singleorder harmonic in the photon energy range of 40-70 eV," Opt. Express, 24(13), 14546-14551 (2016).

[2] L. Poletto, P. Villoresi, F. Frassetto et al., "Time-delay compensated monochromator for the spectral selection of extreme-ultraviolet high-order laser harmonics," Rev. Sci. Instrum., 80(12), 123109 (2009).

[3] S. Bengtsson, and J. Mauritsson, "Ultrafast control and opto-optical modulation of extreme ultraviolet light," J. Phys. B: At. Mol. Opt. Phys., 52(6), 063002 (2019).

[4] M. Schultze, M. Fieß, N. Karpowicz et al., "Delay in photoemission," Science, 328(5986), 1658-1662 (2010).

[5] E. Goulielmakis, Z. Loh, A. Wirth et al., "Real-time observation of valence electron motion," Nature, 466(7307), 739-743 (2010).

[6] J. Ullrich, R. Moshammer, A. Dorn et al., "Recoil-ion and electron momentum spectroscopy: reactionmicroscopes," Rep. Progr. Phys., 66(9), 1463 (2003).

[7] N. Harkema, A. Plunkett, and A. Sandhu, "Tunable high-order frequency mixing for XUV transient absorption and photoelectron spectroscopies," Opt. Express, 27(21), 31053-31061 (2019).

[8] W. Cao, E. R. Warrick, A. Fidler et al., "Noncollinear wave mixing of attosecond XUV and few-cycle optical laser pulses in gas-phase atoms: Toward multidimensional spectroscopy involving XUV excitations," Phys. Rev. A, 94(5), 053846 (2016).

[9] G. Ndabashimiye, S. Ghimire, M. Wu et al., "Solid-state harmonics beyond the atomic limit," Nature, 534(7608), 520-523 (2016).

[10] T. T. Luu, Z. Yin, A. Jain et al., "Extreme-ultraviolet high-harmonic generation in liquids," Nat. Comm., 9(1), $3723(2018)$.

[11] J. Duris, S. Li, T. Driver et al., "Tunable isolated attosecond X-ray pulses with gigawatt peak power from a free-electron laser," Nat. Photon., 14(1), 30-36 (2020).

[12] A. D. Shiner, C. Trallero-Herrero, N. Kajumba et al., "Wavelength scaling of high harmonic generation efficiency," Phys. Rev. Lett., 103(7), (2009).

[13] J. Li, J. Lu, A. Chew et al., "Attosecond science based on high harmonic generation from gases and solids," Nat. Comm., 11(1), 2748 (2020).

[14] S. Mukamel, [Principles of Nonlinear Optical Spectroscopy] Oxford University Press, New York, (1995).

[15] R. W. Boyd, [Nonlinear Optics] Academic Press, (2019).

[16] L. V. Dao, K. A. Tran, and P. Hannaford, "Cascaded four-wave mixing in the XUV region," Opt. Lett., 43(1), 134 (2018).

[17] P. B. Corkum, "Plasma perspective on strong field multiphoton ionization," Phys. Rev. Lett., 71(13), 1994-1997 (1993).

[18] M. Lewenstein, P. Balcou, M. Y. Ivanov et al., "Theory of high-harmonic generation by low-frequency laser fields," Phys. Rev. A, 49(3), 2117-2132 (1994).

[19] A. Gorlach, O. Neufeld, N. Rivera et al., "The quantum-optical nature of high harmonic generation," Nat. Comm., 11(1), 4598 (2020).

[20] T. Popmintchev, M.-C. Chen, A. Bahabad et al., "Phase matching of high harmonic generation in the soft and hard X-ray regions of the spectrum," Proc. Nat. Acad. Sci., 106(26), 10516-10521 (2009).

[21] M. Ammosov, N. Delone, V. Krainov et al., "Tunnel ionization of complex atoms and of atomic ions in an alternating electric field," Sov. Phys. JETP, 64(1191), 26 (1986).

[22] C. Hernández-García, J. A. Pérez-Hernández, J. Ramos et al., "High-order harmonic propagation in gases within the discrete dipole approximation," Phys. Rev. A, 82(3), (2010).

[23] S. Kazamias, F. Weihe, D. Douillet et al., "High order harmonic generation optimization with an apertured laser beam," Eur. Phys. J. D, 21(3), 353-359 (2002).

[24] S. Teichmann, P. Hannaford, and L. V. Dao, "Phase-matched emission of few high-harmonic orders from a helium gas cell," Appl. Phys. Lett., 94(17), 171111 (2009).

[25] L. V. Dao, K. B. Dinh, and P. Hannaford, "Perturbative optical parametric amplification in the extreme ultraviolet," Nat. Comm., 6(1), (2015). 
[26] J. B. Bertrand, H. J. Wörner, H. C. Bandulet et al., "Ultrahigh-Order Wave Mixing in Noncollinear High Harmonic Generation," Physical Review Letters, 106(2), (2011).

[27] L. V. Dao, K. B. Dinh, H. V. Le et al., "Wave-mixing with high-order harmonics in extreme ultraviolet region," Applied Physics Letters, 106(2), 021118 (2015).

[28] K. A. Tran, K. B. Dinh, P. Hannaford et al., "Phase-matched four-wave mixing in the extreme ultraviolet region,” J. Appl. Phys., 124(1), 015901 (2018).

[29] M. Wu, S. Chen, S. Camp et al., "Theory of strong-field attosecond transient absorption," J. Phys. B: At. Mol. Opt. Phys., 49(6), 062003 (2016).

[30] K. B. Dinh, K. A. Tran, P. Hannaford et al., "Four-wave mixing of extreme ultraviolet pulses and infrared pulses for studies of atomic dynamics," J. Opt. Soc. America B, 36(11), 3046-3052 (2019).

[31] K. A. Tran, K. B. Dinh, T. H. Chau et al., "Dynamics of free electron wave packets with coherent extreme ultraviolet four-wave mixing fields," Optik, 225, 165833 (2021).

[32] D. Bleiner, The Science and Technology of X-ray Lasers: A 2020 Update Proc. SPIE 11886-00 (2021) 